Research Article

\title{
Strategic WIP Inventory Positioning for Make-to-Order Production with Stochastic Processing Times
}

\author{
Jingjing Jiang and Suk-Chul Rim \\ Department of Industrial Engineering, Ajou University, Suwon 16499, Republic of Korea \\ Correspondence should be addressed to Suk-Chul Rim; scrim@ajou.ac.kr
}

Received 15 June 2017; Revised 7 September 2017; Accepted 10 October 2017; Published 28 November 2017

Academic Editor: Emilio Jiménez Macías

Copyright (c) 2017 Jingjing Jiang and Suk-Chul Rim. This is an open access article distributed under the Creative Commons Attribution License, which permits unrestricted use, distribution, and reproduction in any medium, provided the original work is properly cited.

\begin{abstract}
It is vital for make-to-order manufacturers to shorten the lead time to meet the customers' requirements. Holding work-in-process (WIP) inventory at more stations can reduce the lead time, but it also brings about higher inventory holding cost. Therefore, it is important to seek out the optimal set of stations to hold WIP inventory to minimize the total inventory holding cost, while meeting the required due date for the final product at the same time. Since the problem with deterministic processing times at the stations has been addressed, as a natural extension, in this study, we address the problem with stochastic processing times, which is more realistic in the manufacturing environment. Assuming that the processing times follow normal distributions, we propose a solution procedure using genetic algorithm.
\end{abstract}

\section{Introduction}

Nowadays, the manufacturing environment has shifted from traditional mass production to small order production, because the development of information technology has resulted in an explosion of product types, shorter product life cycles, a wider variety of customer requirements, and faster changes, which make demand forecasts more difficult.

In 1964, the material requirements planning (MRP) was developed by Joesph Orlicky, and in the following two decades various investigations on MRP had been conducted. However, due to the lower demand forecast accuracy, the performance of traditional MRP became barely satisfactory. Therefore, a new type of MRP called Demand Driven MRP (DDMRP) was introduced in 2011 by Ptak and Smith [1], which enables a manufacturer to respond more closely to the actual market requirements and promotes better and quicker decision and actions in the planning and execution stage. In traditional MRP inventory planning, most efforts are focused on solving two questions: how to hold inventory and when to reorder goods. However, in DDMRP, the focus is on where to position the inventory, because "how" question is meaningless until we can answer the "where" question appropriately. DDMRP has its own logical and effective approach to answer the "how to hold" question. Determining the best inventory locations can solve the problems of satisfying inventory performance and delivery performance.

One of the most important competitive factors in maketo-order manufacturing is to deliver the product to the customer within the due time that the customer requires. In order to shorten the delivery lead time, WIP inventories need to be held at major processes. Meanwhile, the total inventory holding cost should be minimized to decrease the financial burden in today's cost-competitive manufacturing environment. It is noteworthy that, for some stations, holding inventory does not contribute to reducing the lead time of final product at all due to the BOM structure and processing times. Hence, determining the optimal set of stations to hold WIP inventory is a complicated and interesting problem.

This problem, namely, the strategic inventory positioning (SIP) problem, has been studied by many researchers. Whybark and Yang [2] proposed a controlled simulation experiment to decide where to place inventory to achieve the best service level. Simpson Jr. [3] used the "all or nothing" policy to decide whether to place the inventory or not in some points in serial line system. Graves and Willems [4-6] extended Simpson Jr. [3] model to assembly, distribution, and spanning 
tree network structures. Lesnaia [7] considered the service time as a stochastic model instead of a deterministic model assumed by Graves and Willems [5]. Magnanti et al. [8] solved the inventory positioning problem at production/assembly stages of components in an acyclic supply chain network structure which is not a spanning tree network structure. Many other researchers studied the inventory positioning problem using different methods such as Kaminsky and Kaya [9], Inderfurth [10], Alderson [11], and Georage et al. [12]. We also have studied SIP problem for simple and general BOM using actively synchronized replenishment (ASR) lead time proposed in the DDMRP [13, 14], in which we present mathematical models and genetic algorithm to solve SIP problems, assuming that the processing times are all deterministic. However, it will be more realistic in most cases to consider the processing times to be stochastic due to various factors such as defectives, reprocessing, equipment setup, and/or calibration time variations. In this paper we address this stochastic SIP problem and present a solution procedure for simple BOM case.

This paper is organized as follows: Section 2 describes the SIP problem and introduces the ASRLT, buffer profiles, and notations; Section 3 presents a genetic algorithm-based solution procedure and numerical examples; finally, the conclusion and potential future research issues are addressed in Section 4.

\section{The Strategic Inventory Positioning Problem}

In the authors' earlier works $[13,14]$, the concept of a new lead time has been introduced, namely, actively synchronized replenishment lead time (ASRLT), which was proposed by Ptak and Smith [1], in which ASRLT is defined as "the longest unprotected or unbuffered sequence in the BOM for a particular parent." The strategic inventory positioning (SIP) problem is to determine the locations which hold WIP inventory in order to meet the lead time the customer requires for the final product, while minimizing the total inventory holding cost. The stochastic SIP problem is the SIP problem where processing times at the nodes follow a certain probability distribution. In this paper, we assume the processing times follow normal distributions.

2.1. Notations. For consistency, we will use in this paper the same $\mathrm{BOM}$ and notations as used in the previous papers $[13$, 14] as follows:

$\operatorname{part}_{i, j}$ : $j$ th part counted from the left in the $i$ th level of the BOM

$p_{i, j}$ : the number counted from the left in the BOM of the parent part of part ${ }_{i, j}$ (e.g., in Figure 1, $p_{3,2}=2, p_{3,4}$ $=3$ )

$r_{i, j}:$ required quantity to make unit immediate parent of part $_{i, j}$ $a_{i, j}:$ ASRLT of part $_{i, j}$

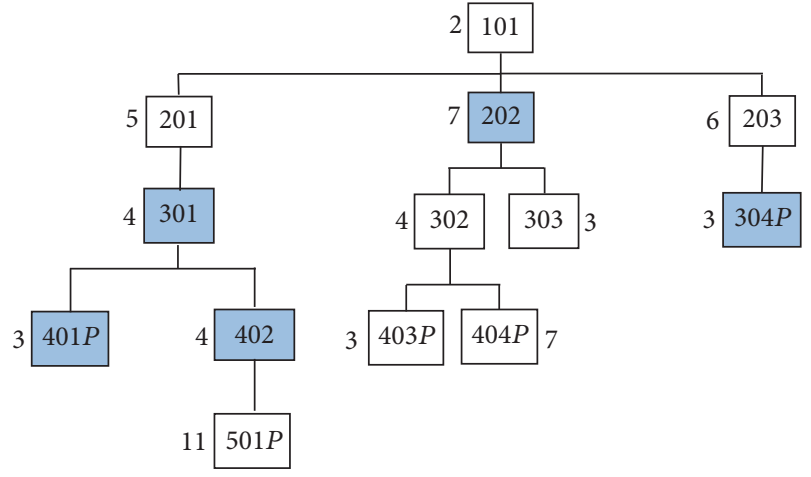

Figure 1: An example of simple bill of materials.

$\mathrm{pr}_{i, j}$ : processing time distribution of part $_{i, j}$ that follows a normal distribution with mean $\mu_{i, j}$ and variance $\sigma_{i, j}{ }^{2}$

$n_{i, j}$ : required quantity of part $_{i, j}$ to make unit end product

$s_{i, j}$ : set of numbers counted from the left in the BOM of the subcomponents of part $_{i, j}$ (e.g., in Figure 1, $s_{2,2}$ $\left.=\{2,3\} ; s_{3,2}=\{3,4\}\right)$

$\operatorname{adu}_{i, j}$ : average daily usage of part $_{i, j}$

$c_{i, j}$ : annual inventory cost of part $_{i, j}$

$\mathrm{ai}_{i, j}$ : average inventory quantity of part $_{i, j}$

$v_{i, j}$ : unit price of part $_{i, j}$

$\operatorname{ltp}_{i, j}$ : percentage usage of part $_{i, j}$ over ASR lead time

$\mathrm{vp}_{i, j}$ : percentage of red zone base of part $_{i, j}$ that red zone safety accounts for

$h$ : annual inventory holding cost rate

$u$ : average daily usage of the end product

$Q$ : lead time for the end product required by the customer (i.e., service time)

$y_{i, j}$ : yellow zone quantity of part $_{i, j}$

$g_{i, j}$ : green zone quantity of part $_{i, j}$

$\mathrm{rb}_{i, j}$ : red zone base quantity of part $\mathrm{p}_{i, j}$

$\mathrm{rs}_{i, j}$ : red zone safety quantity of part $\mathrm{p}_{i, j}$.

Decision variables:

$$
x_{i, j}= \begin{cases}1 & \text { if } \text { part }_{i, j} \text { has inventory } \\ 0 & \text { if } \text { part }_{i, j} \text { has no inventory. }\end{cases}
$$

In our earlier papers $[13,14]$, all the processing times are assumed to be deterministic, so the minimum total inventory holding cost is also a deterministic value. In this paper, however, the processing times are assumed to follow normal distributions. So we generate $M$ sets of random processing times to obtain $M$ different ASRLTs of the end product and corresponding minimum total inventory holding costs. Then 
the final minimum total inventory holding cost is the average of $M$ different minimum total inventory holding costs. We define that a solution $X=\left\{x_{i j}\right\}$ is feasible if the probability $\alpha$ that ASR lead time of end product for $X$ is not greater than $Q$ is not smaller than $\alpha_{0}$, where $0<\alpha_{0}<1$, but close to 1 ; for example, $\alpha_{0}=0.95$. Equation (2) is the total inventory holding cost once the inventory location is determined as $X=\left\{x_{i j}\right\}$. Equation (3) is the annual inventory holding cost of part $t_{i, j}$.

$$
\begin{aligned}
\mathrm{TC} & =\sum_{\forall i} \sum_{\forall j} c_{i, j} \cdot x_{i, j} \\
c_{i, j} & =v_{i, j} \cdot \mathrm{ai}_{i, j} \cdot h .
\end{aligned}
$$

2.2. Inventory Control Scheme in DDMRP. In their book [1], Ptak and Smith proposed an inventory control scheme as follows: there are five color zones that comprise the total buffer: green zone represents an inventory position that requires no actions; yellow zone represents an inventory position that requires replenishment; both red zone base and red zone safety represent an inventory position that requires special attention. The average inventory quantity can be calculated as the total quantity of red zone plus half of green zone, as (4). The magnitude of yellow zone is determined as the average usage multiplied by ASR lead time of part pas $_{j}$ as (5).

$$
\begin{aligned}
\mathrm{ai}_{i, j} & =0.5 \cdot g_{i, j}+\mathrm{rb}_{i, j}+\mathrm{rs}_{i, j} \\
y_{i, j} & =\mathrm{adu}_{i, j} \cdot a_{i, j} .
\end{aligned}
$$

The magnitudes of red zone base and green zone are determined as the percentage usage of part $_{i, j}$ over ASR lead time multiplied by yellow zone quantity, as (6) and (7). The magnitude of red zone safety is determined as percentage of red zone base of part $_{i, j}$ that red zone safety accounts for, multiplied by red zone base, as (8). Table 1 shows the recommended ranges of impact factor for the green and red zone bases, according to the relative length of lead time of the part. Table 2 shows how red zone safety is sized according to the demand variability. Note that minimum order quantity (MOQ) is not considered in this paper.

$$
\begin{aligned}
\mathrm{rb}_{i, j} & =\operatorname{ltp}_{i, j} \cdot y_{i, j} \\
g_{i, j} & =\operatorname{ltp}_{i, j} \cdot y_{i, j} \\
\mathrm{rs}_{i, j} & =\mathrm{vp}_{i, j} \cdot \mathrm{rb}_{i, j} .
\end{aligned}
$$

2.3. Mathematical Modeling. Using the above notations and inventory control scheme, we model the SIP problem mathematically as follows:

$$
\begin{aligned}
& n_{i, j}=r_{i, j} \cdot n_{i-1, p_{i, j}} \\
& \operatorname{adu}_{i, j}=\mu \cdot n_{i, j} \\
& a_{i, j} \\
& = \begin{cases}\max _{m \in s_{i, j}}\left[\left(1-x_{i+1, m}\right) a_{i+1, m}+t_{i, j}\right], & \text { if } s_{i, j} \neq \emptyset \\
a_{i, j}=t_{i, j}, & \text { if } s_{i, j}=\emptyset: \text { end node. }\end{cases}
\end{aligned}
$$

TABLE 1: Recommended impact ranges for green and red zone base.

Long lead time

Medium lead time

Short lead time

TABLE 2: Recommended impact ranges for red zone safety.
High variability

Medium variability

Low variability
$20-40 \%$ usage over LT

$41-60 \%$ usage over LT

$61-100 \%$ usage over LT
We want to find a set $X=\left\{x_{i j}\right\}$ to minimize the average total inventory holding cost, while the ASRLT of the end product is no longer than the customer's requirement $Q$ with probability $\alpha \geq \alpha_{0}$, where $0<\alpha_{0}<1$, but close to 1 ; for example, $\alpha_{0}=0.95$. From (2) to (9), the optimal solution to the SIP problem can be obtained by solving the following mathematical model:

$$
\begin{gathered}
\text { Minimize average total cost: } \frac{\sum \sum_{\forall i} \sum_{\forall j} v_{i, j} \cdot h \cdot \mathrm{ai}_{i, j} \cdot x_{i, j}}{M} \\
\text { st. } \quad \mathrm{ai}_{i, j}=\left(1.5+\mathrm{vp}_{i, j}\right) \cdot \operatorname{ltp}_{i, j} \cdot \mathrm{adu}_{i, j} \cdot a_{i, j} \\
\operatorname{adu}_{i, j}=u \cdot n_{i, j} \\
n_{i, j}=r_{i, j} \cdot n_{i-1, p_{i, j}} \\
a_{i, j}= \begin{cases}\max _{m \in s_{i, j}}\left[\left(1-x_{i+1, m}\right) a_{i+1, m}+t_{i, j}\right], & \text { if } s_{i, j} \neq \emptyset \\
a_{i, j}=t_{i, j}, & \text { if } s_{i, j}=\emptyset: \text { end node }\end{cases} \\
a_{1,1} \leq Q \\
\alpha \geq \alpha_{0},
\end{gathered}
$$

where $M$ is the number of replications.

The above model is not easy to solve because it is not a LP problem. In the next section, we will present the genetic algorithm-based solution procedure for the problem.

\section{Solution Procedure and Numerical Example}

In the deterministic SIP problem addressed in our earlier papers $[13,14]$, the ASR lead time of the end product $(W)$ can be determined as a value, depending on the binary solution $X=\left\{x_{i j}\right\}$, since all processing times are deterministic. So a solution $X=\left\{x_{i j}\right\}$ is feasible if $W$ is not greater than $Q$, the lead time the customer requires. However, in the stochastic SIP problem we address in this paper, since different processing times will be generated every time for each node, $W$ will also be different even for a particular solution $X=\left\{x_{i j}\right\}$. So for the stochastic SIP we define that a solution $X=\left\{x_{i j}\right\}$ is feasible if $W$ is not greater than $Q$ with probability $\alpha \geq \alpha_{0}$, where $0<\alpha_{0}<1$, but close to 1 ; for example, $\alpha_{0}=0.95$.

To implement this in the solution procedure, for a particular solution $X=\left\{x_{i j}\right\}$ and parameters $\mu_{i, j}$ and $\sigma_{i, j}{ }^{2}$, representing the mean and variance of the normally distributed processing time of part $_{i, j}$, respectively, we generate a set of random processing times $\left\{t_{i, j}\right\}$ for all parts and compute $W$ by the genetic algorithm that we used in the earlier paper 


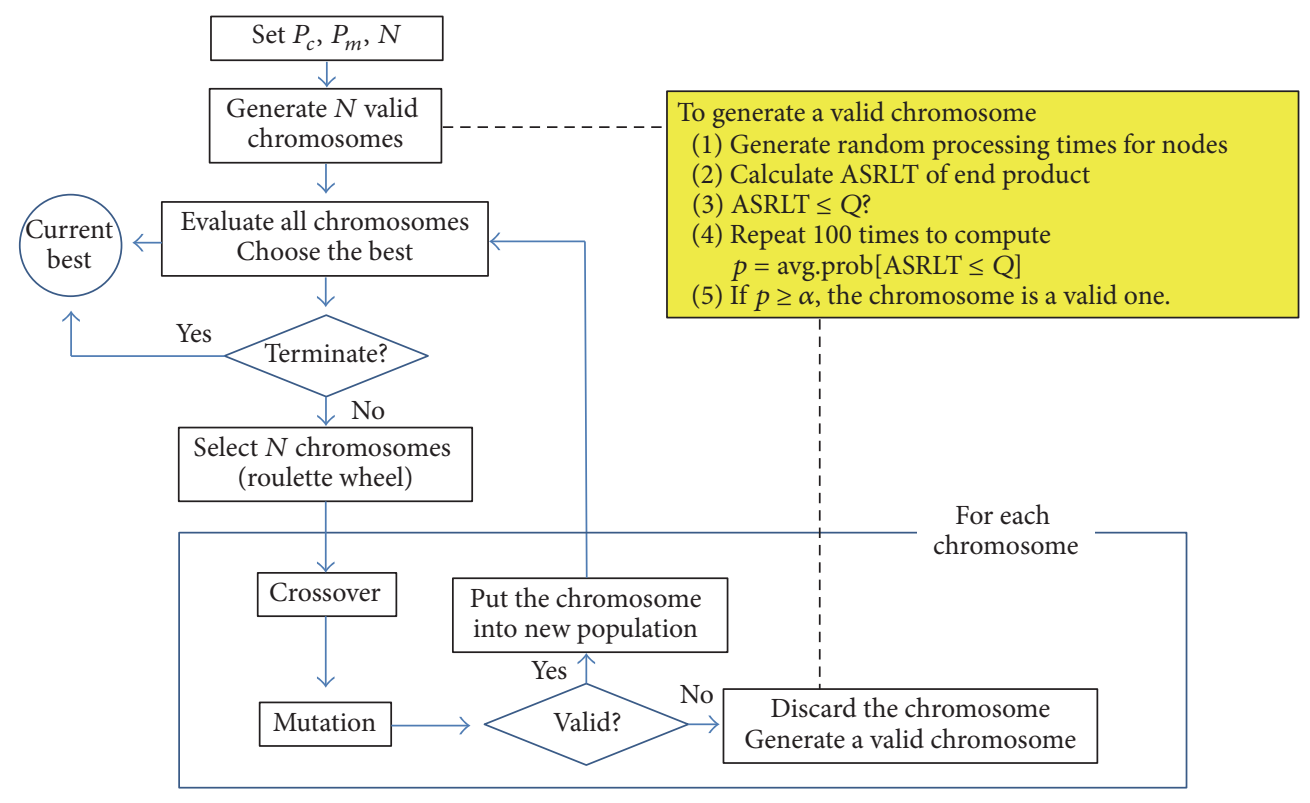

FIgURE 2: The flow chart of GA-based solution procedure.

for deterministic SIP problem. Repeat this for 100 times to compute the probability $(\alpha)$ that the ASRLT is not greater than $Q$. If $\alpha$ is greater than or equal to a given value $\alpha_{0}$, then the solution $X=\left\{x_{i j}\right\}$ is regarded as a valid solution. Then the total inventory holding cost of $X=\left\{x_{i j}\right\}$ is computed as the average of 100 inventory holding costs.

As shown in Figure 2, the solution procedure is that we generate $N$ valid chromosomes, evaluate all chromosomes by calculating the average total inventory holding cost, and keep the best one. We select chromosomes by roulette wheel rule. Once two chromosomes are selected, they undergo crossover and mutate. Then new population including $N$ chromosomes is created. We test the validity of each chromosome, discard invalid chromosomes, and generate new valid chromosomes to be put to the population so as to keep the number of chromosomes in the populations unchanged to be $N$. Then we evaluate all chromosomes and choose the best one in this new population. If this best one is better than the current best, then replace the current best with the new best one (called elitism rule). We set the termination condition as running several generations, usually depending on the number of parts in the BOM. The more the parts in the BOM are, the more the generation run is required. In order to evaluate the performance of the proposed genetic algorithm-based solution procedure, two numerical examples are given as follows.

3.1. Small BOM Case. Consider a small BOM comprising 13 parts, as shown in Figure 1. The parameters are given in Table 3 . We set the same variability percentage (vp) as $50 \%$ for all parts. The lead time percentage (ltp) is 30\%, 50\%, and $80 \%$ for long, medium, and short lead time, respectively. $\alpha_{0}$ is set as $95 \%$. We set the crossover rate $P_{c}$ as 0.8 and the mutation rate $P_{m}$ as 0.1 . For small instances, we set the population size $N$ as 80 . The iteration will stop after 100 generations.
In order to evaluate the performance of the proposed GAbased procedure, the result is compared with that obtained from enumeration. For each $X=\left\{x_{i j}\right\}$, we generate $M=100$ sets of processing times. Among the valid solutions out of $2^{13}$ possible solutions, we choose the twenty smallest average total inventory holding costs using enumeration, as shown in Table 4.

Table 5 shows the optimal total inventory holding cost of 10 replications of the proposed solution procedure. In the previous problem with deterministic processing time for each node, the optimal total inventory holding cost obtained using GA can be matched with the ranking using enumeration. However, in the stochastic SIP problem, it is impossible to match the optimal total inventory holding cost from the proposed procedure with the ranking list obtained from enumeration. Therefore, we run the proposed procedure for 10 replications and list the result, as shown in Table 5, so that we can compare the result with the ranking list obtained from enumeration. Note that the largest total cost $(1,671.78)$ out of the 10 replications in Table 5 is approximately same as the 5 th smallest total cost from enumeration, which indicates that the performance of the proposed procedure is satisfactory. Each replication takes about 30 seconds in the McIntosh PC.

3.2. Large BOM Case. In this example, we consider a BOM comprising 50 parts, which means that there are $2^{50}$ possible solutions. Table 6 lists the parameters in which vp, lvp, $h, P_{c}$, and $P_{m}$ assume the same values as those in small BOM case, but $Q$ is set as 16 . For the problems of same size, computation time depends on the population size $(N)$ and the number of iterations that we set as the termination condition. We set the program to stop after 200 iterations. We can certainly increase the number of iterations, but due to the increasing computation time, we think that 200 iterations for each replication will be enough to show how the objective function 
TABLE 3: Parameters for small BOM case.

\begin{tabular}{|c|c|c|c|c|c|c|c|c|c|c|c|c|c|}
\hline Part & 1 & 2 & 3 & 4 & 5 & 6 & 7 & 8 & 9 & 10 & 11 & 12 & 13 \\
\hline$\mu$ & 2 & 5 & 7 & 6 & 4 & 4 & 3 & 3 & 3 & 4 & 3 & 7 & 11 \\
\hline$\sigma$ & 0.4 & 0.9 & 1.1 & 0.8 & 0.6 & 0.5 & 0.9 & 0.7 & 0.8 & 0.9 & 0.4 & 1.3 & 2.0 \\
\hline$p$ & 0 & 1 & 1 & 1 & 1 & 2 & 2 & 3 & 1 & 1 & 2 & 2 & 2 \\
\hline$v$ & 700 & 120 & 250 & 100 & 65 & 80 & 110 & 40 & 24 & 16 & 21 & 27 & 9 \\
\hline Q & 10 & & & & & & & & & & & & \\
\hline$h$ & 0.25 & & & & & & & & & & & & \\
\hline $\mathrm{vp}$ & 0.5 & & & & & & & & & & & & \\
\hline \multirow[t]{3}{*}{ ltp } & Lon & time & + days) & & & & & $30 \%$ & & & & & \\
\hline & $\mathrm{Mec}$ & lead $t$ & (11-25 & & & & & $50 \%$ & & & & & \\
\hline & Sho & d time & 10 days & & & & & $80 \%$ & & & & & \\
\hline$\alpha_{0}$ & $95 \%$ & & & & & & & & & & & & \\
\hline
\end{tabular}

TABLE 4: Twenty smallest average total costs obtained from enumeration.

\begin{tabular}{lc}
\hline Rank & Total cost \\
\hline 1 & 1492.95 \\
2 & 1584.6 \\
3 & 1607.17 \\
4 & 1659.8 \\
5 & 1687.71 \\
6 & 1696.45 \\
7 & 1702.6 \\
8 & 1707.46 \\
9 & 1722.15 \\
10 & 1763.8 \\
11 & 1773.07 \\
12 & 1782.1 \\
13 & 1785.3 \\
14 & 1785.87 \\
15 & 1796.09 \\
16 & 1812.1 \\
17 & 1816.09 \\
18 & 1828.61 \\
19 & 1831.39 \\
20 & 1838.2 \\
\hline
\end{tabular}

TABLE 5: The results of 10 replications obtained from the proposed procedure.

\begin{tabular}{lc}
\hline Number & Total cost \\
\hline 1 & 1671.78 \\
2 & 1665.13 \\
3 & 1529.41 \\
4 & 1457.4 \\
5 & 1611.37 \\
6 & 1551.59 \\
7 & 1489.03 \\
8 & 1601.44 \\
9 & 1493.87 \\
10 & 1597.08 \\
\hline
\end{tabular}

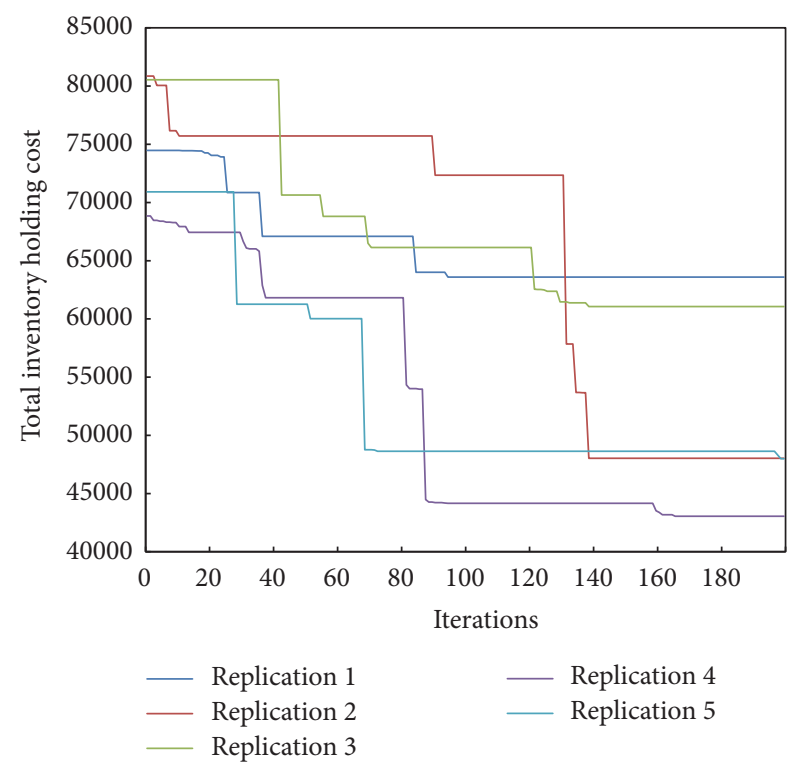

FIGURE 3: Decrease of objective function values for 5 replications for large BOM case.

values decrease as the iteration progresses. $N$ is set as 300 . We run 5 replications, each of which takes about 13 minutes in the same PC. We chose the number of replications as 5 arbitrarily. We can certainly increase the number of replications, but displaying too many lines in a figure will make the figure messy, without adding any implication.

Note that we cannot explicitly demonstrate the performance of the proposed solution procedure for a problem with large BOM of 50 nodes since we cannot enumerate the $2^{50}$ possible solutions. Instead we show the decrease of objective function values of five replications in Figure 3. Recall that the best result obtained from GA when it terminates does not guarantee being optimal.

\section{Conclusion}

In this paper, we address the problem of determining the stations to hold work-in-process inventory in order to reduce 
TABLE 6: Parameters for large BOM case.

\begin{tabular}{|c|c|c|c|c|c|}
\hline Part & $\mu$ & $\sigma$ & $r$ & $p$ & $v$ \\
\hline 1 & 2 & 0.2 & 1 & 0 & 1200 \\
\hline 2 & 5 & 0.3 & 1 & 1 & 900 \\
\hline 3 & 7 & 2 & 1 & 1 & 890 \\
\hline 4 & 6 & 1.2 & 1 & 1 & 910 \\
\hline 5 & 4 & 0.8 & 2 & 1 & 860 \\
\hline 6 & 4 & 0.7 & 3 & 1 & 880 \\
\hline 7 & 3 & 0.4 & 2 & 1 & 800 \\
\hline 8 & 3 & 0.3 & 1 & 1 & 780 \\
\hline 9 & 3 & 0.3 & 2 & 2 & 790 \\
\hline 10 & 4 & 0.4 & 3 & 2 & 750 \\
\hline 11 & 3 & 0.4 & 2 & 3 & 690 \\
\hline 12 & 5 & 0.4 & 3 & 3 & 660 \\
\hline 13 & 5 & 0.4 & 4 & 4 & 600 \\
\hline 14 & 4 & 0.3 & 3 & 4 & 640 \\
\hline 15 & 2 & 0.2 & 1 & 4 & 700 \\
\hline 16 & 4 & 0.3 & 3 & 1 & 660 \\
\hline 17 & 6 & 0.9 & 2 & 1 & 580 \\
\hline 18 & 3 & 0.5 & 1 & 2 & 550 \\
\hline 19 & 3 & 0.4 & 1 & 3 & 570 \\
\hline 20 & 5 & 0.8 & 2 & 3 & 600 \\
\hline 21 & 7 & 1.1 & 3 & 4 & 540 \\
\hline 22 & 6 & 1.0 & 2 & 4 & 510 \\
\hline 23 & 3 & 0.5 & 2 & 4 & 590 \\
\hline 24 & 4 & 0.5 & 1 & 5 & 400 \\
\hline 25 & 3 & 0.5 & 1 & 6 & 490 \\
\hline 26 & 3 & 0.4 & 1 & 7 & 480 \\
\hline 27 & 5 & 0.7 & 2 & 7 & 520 \\
\hline 28 & 4 & 0.4 & 2 & 8 & 400 \\
\hline 29 & 3 & 0.5 & 1 & 9 & 480 \\
\hline 30 & 2 & 0.2 & 3 & 9 & 490 \\
\hline 31 & 5 & 0.6 & 1 & 1 & 460 \\
\hline 32 & 4 & 0.6 & 1 & 1 & 500 \\
\hline 33 & 3 & 0.3 & 3 & 3 & 470 \\
\hline 34 & 3 & 0.3 & 2 & 3 & 300 \\
\hline 35 & 5 & 0.6 & 2 & 5 & 380 \\
\hline 36 & 4 & 0.4 & 2 & 8 & 330 \\
\hline 37 & 3 & 0.4 & 3 & 8 & 340 \\
\hline 38 & 4 & 0.3 & 1 & 12 & 350 \\
\hline 39 & 6 & 0.6 & 1 & 12 & 360 \\
\hline 40 & 4 & 0.4 & 3 & 15 & 310 \\
\hline 41 & 4 & 0.5 & 2 & 2 & 330 \\
\hline 42 & 4 & 0.5 & 2 & 3 & 290 \\
\hline 43 & 3 & 0.3 & 2 & 3 & 270 \\
\hline 44 & 3 & 0.4 & 2 & 5 & 260 \\
\hline 45 & 2 & 0.2 & 1 & 7 & 270 \\
\hline 46 & 5 & 0.5 & 1 & 7 & 270 \\
\hline 47 & 3 & 0.4 & 3 & 9 & 100 \\
\hline 48 & 2 & 0.4 & 2 & 9 & 110 \\
\hline 49 & 3 & 0.6 & 4 & 5 & 50 \\
\hline 50 & 5 & 0.9 & 2 & 7 & 30 \\
\hline
\end{tabular}

the production lead time of the end product to the one the customer requires, while minimizing the total average inventory cost in make-to-order manufacturing. As an extension of the earlier work of the authors, where processing times are assumed to be constant at each station, in this paper we consider the stochastic version of the problem in which processing times follow certain probability distribution, namely, stochastic strategic inventory positioning (SIP) problem. This will be more realistic than assuming deterministic processing times. We formulate the stochastic SIP problem mathematically and present a genetic algorithmbased solution procedure. Numerical examples show the validity of the proposed solution procedure.

In most MTO manufacturing sites, it is not unusual to find that almost all stations maintain WIP inventory, mainly due to natural human behavior of protection. However, having WIP inventory at some stations does not contribute at all to reducing the total manufacturing lead time of end product because of the BOM structure and lead time of other parts in the BOM. To minimize the WIP inventory cost, such stations should not have WIP inventory. The result of this paper will help determine strategically where to have and where not to have WIP inventory. We use inventory control scheme of DDMRP to compute the average inventory at a station. For further research, one may study the case where some parts are used more than once in the BOM.

\section{Conflicts of Interest}

The authors declare that there are no conflicts of interest regarding the publication of this paper.

\section{Acknowledgments}

This research was sponsored by Jeongseok Foundation. The authors would like to express their thanks to Jeongseok Foundation for support during the research.

\section{References}

[1] C. Ptak and C. Smith, Orlicky's Material Requirements Planning 3/E, McGraw-Hill Education, New York, NY, USA, 2011.

[2] D. C. Whybark and S. Yang, "Positioning inventory in distribution systems," International Journal of Production Economics, vol. 45, no. 1-3, pp. 271-278, 1996.

[3] K. F. Simpson Jr., "In-process inventories," Operations Research, vol. 6, no. 6, pp. 863-873, 1958.

[4] S. C. Graves and S. P. Willems, "Strategic safety stock placement in supply chains," in Proceedings of the MSOM Conference, Hanover, NH, USA, 1996.

[5] S. C. Graves and S. P. Willems, "Optimizing strategic safety stock placement in supply chains," Manufacturing \& Service Operations Management, vol. 2, no. 1, pp. 68-83, 2000.

[6] S. C. Graves and S. P. Willems, "Strategic inventory placement in supply chains: nonstationary demand," Manufacturing \& Service Operations Management, vol. 10, no. 2, pp. 278-287, 2008. 
[7] E. Lesnaia, Optimizing safety stock placement in general network supply chains [Ph.D. thesis], MIT Operations Research Center, Cambridge, UK, 2004.

[8] T. L. Magnanti, Z. M. Shen, J. Shu, D. Simchi-Levi, and C.-P. Teo, "Inventory placement in acyclic supply chain networks," Operations Research Letters, vol. 34, no. 2, pp. 228-238, 2006.

[9] P. Kaminsky and O. Kaya, "Inventory positioning, scheduling and lead-time quotation in supply chains," International Journal of Production Economics, vol. 114, no. 1, pp. 276-293, 2008.

[10] K. Inderfurth, "Safety stock optimization in multi-stage inventory systems," International Journal of Production Economics, vol. 24, no. 1-2, pp. 103-113, 1991.

[11] W. Alderson, "Marketing efficiency and the principle of postponement," in Marketing Behavior and Executive Action, pp. 423-427, 1957.

[12] I. Georage, G. Prastacos, and G. Skintzi, "Inventory positioning in multiple product supply chains," Annals of Operations Research, vol. 126, pp. 195-213, 2004.

[13] S.-C. Rim, J. J. Jiang, and C. J. Lee, "Strategic inventory positioning for MTO manufacturing using ASR lead time," in Logistics Operations, Supply Chain Management and Sustainability, P. Golinska, Ed., EcoProduction, pp. 441-456, Springer, Basel, Switzerland, 2014.

[14] J. Jiang and S.-C. Rim, "Strategic Inventory Positioning in BOM with Multiple Parents Using ASR Lead Time," Mathematical Problems in Engineering, vol. 2016, Article ID 9328371, 2016. 


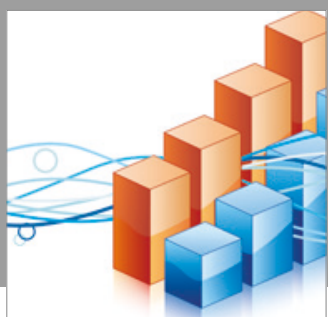

Advances in

Operations Research

vatersals

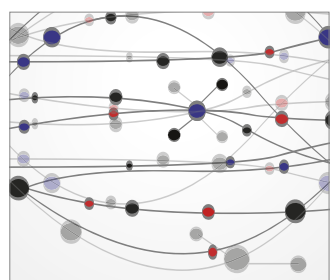

\section{The Scientific} World Journal
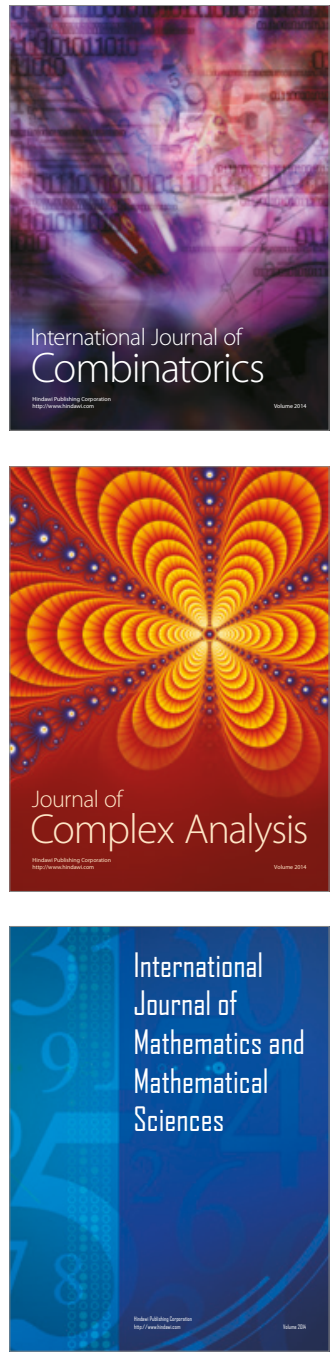
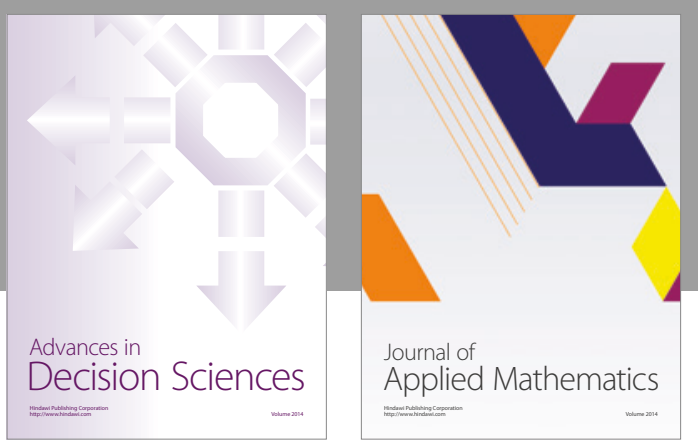

Algebra

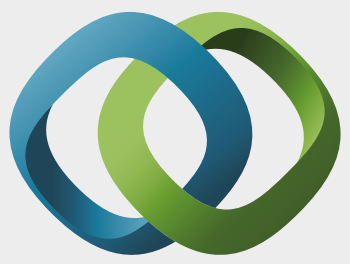

\section{Hindawi}

Submit your manuscripts at

https://www.hindawi.com
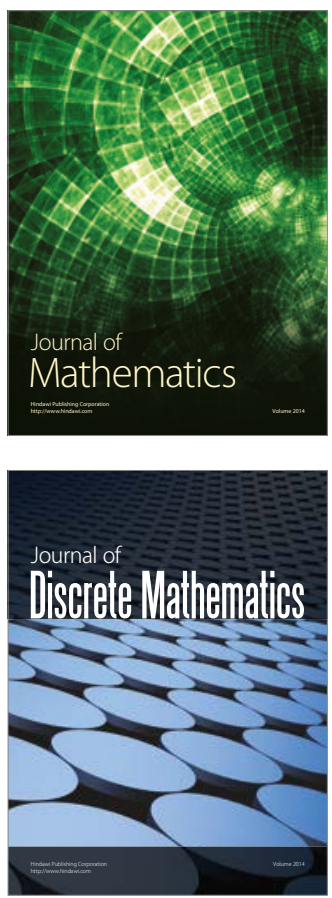

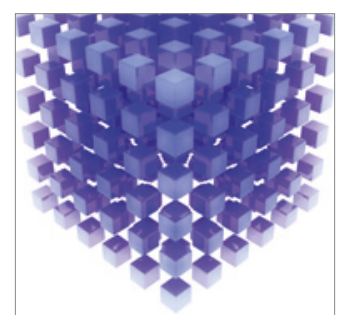

Mathematical Problems in Engineering
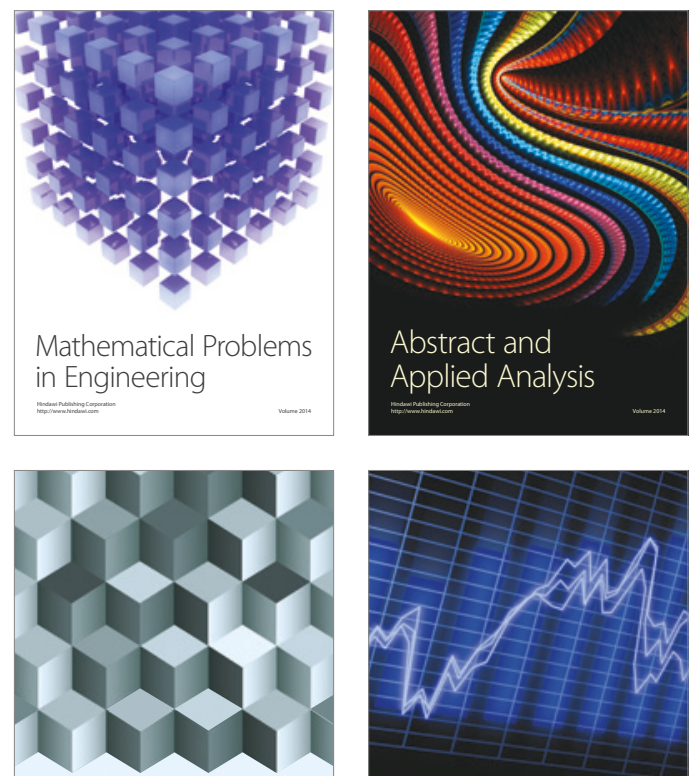

Journal of

Function Spaces

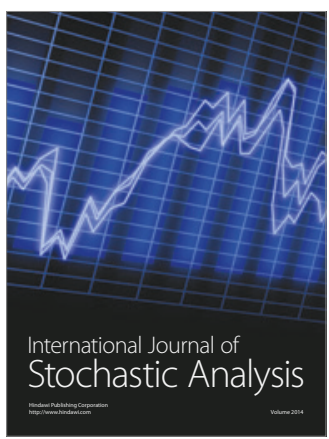

Probability and Statistics
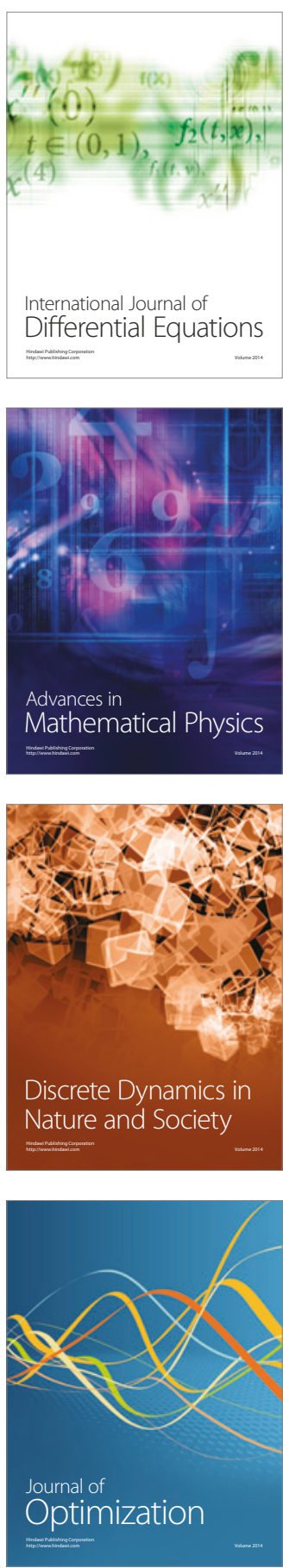